\title{
3 Anhang
}

\subsection{Untersuchungsmaterial Gesamtkorpus}

\subsubsection{Lokalpolitik}

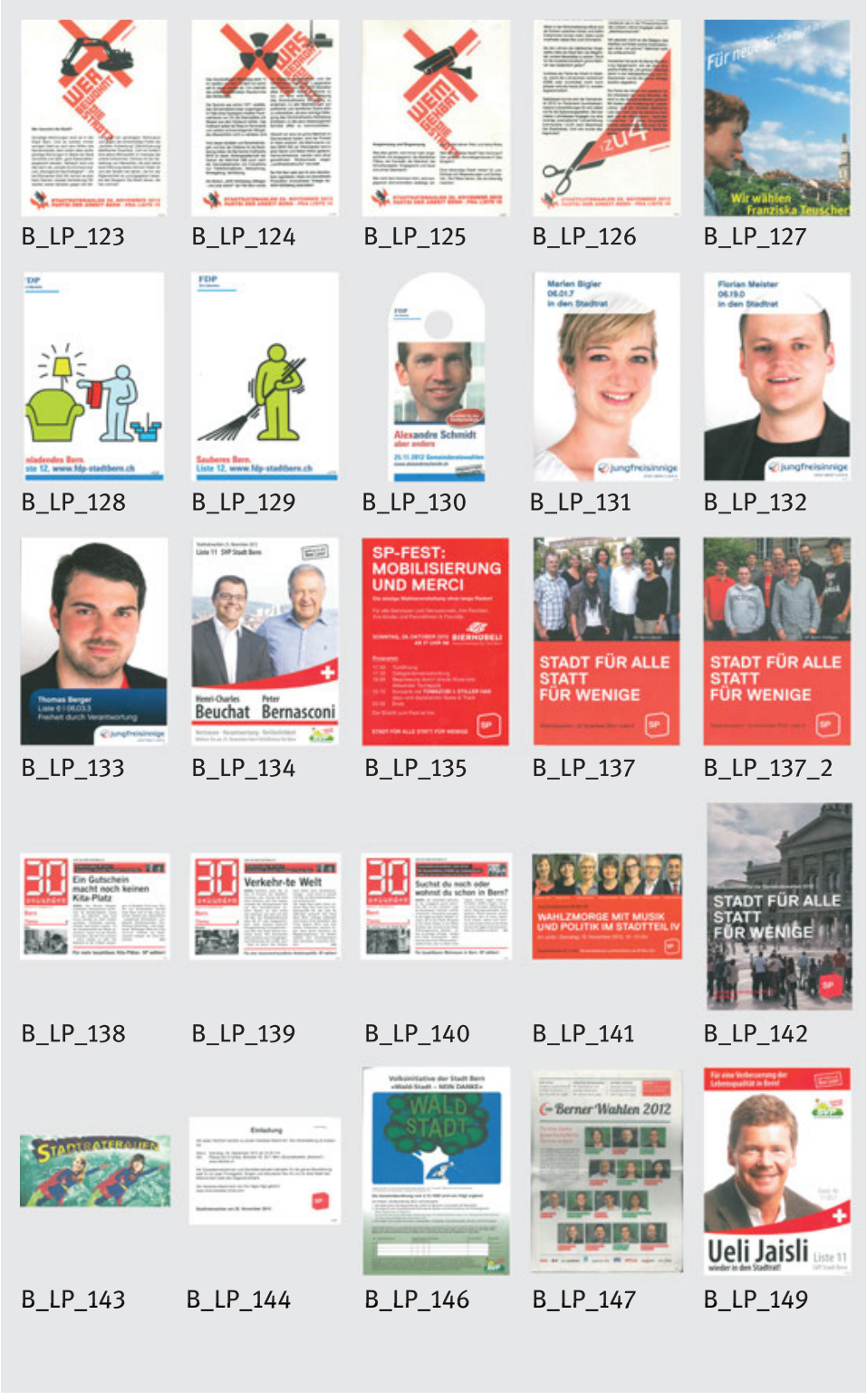




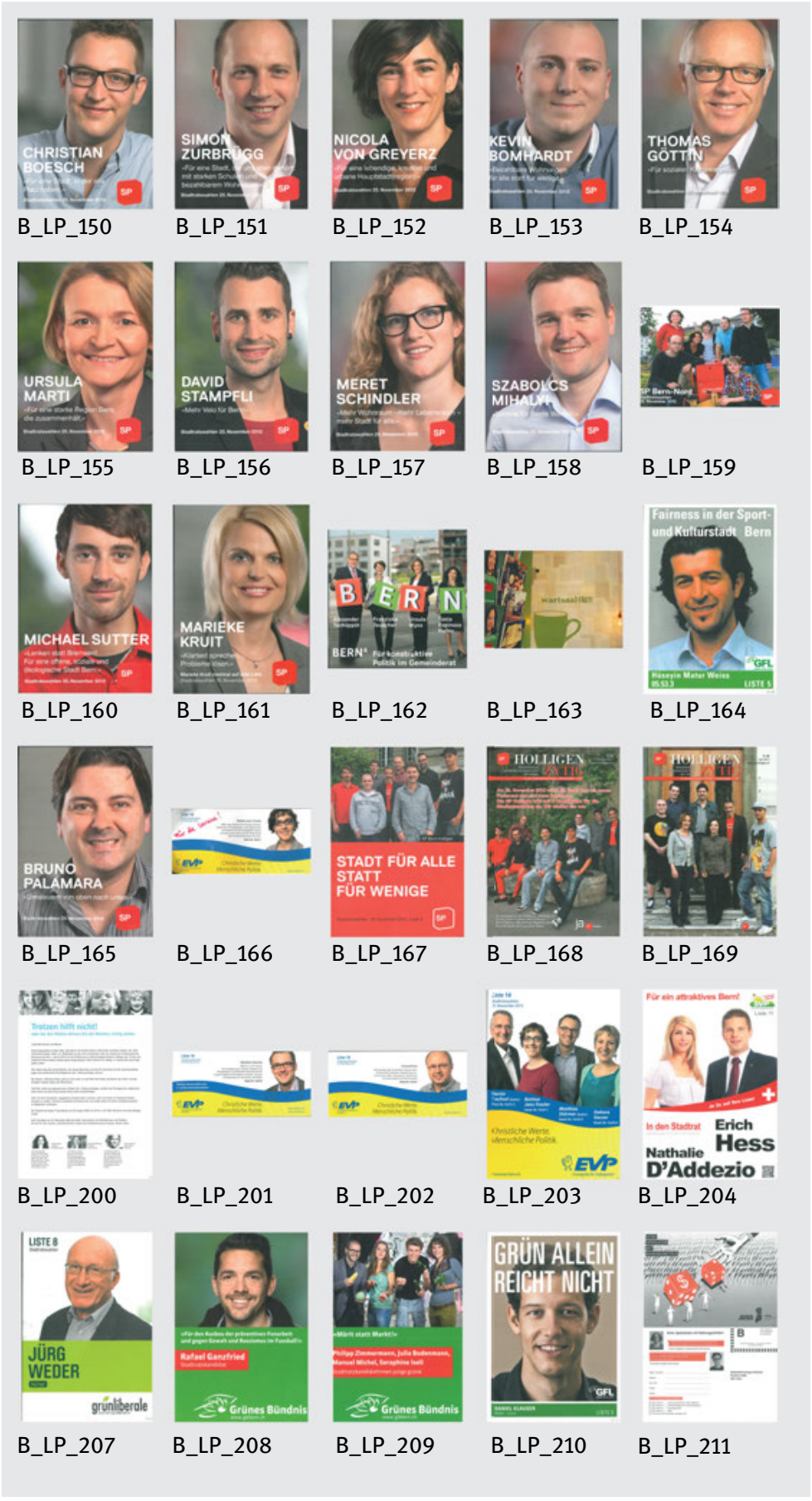




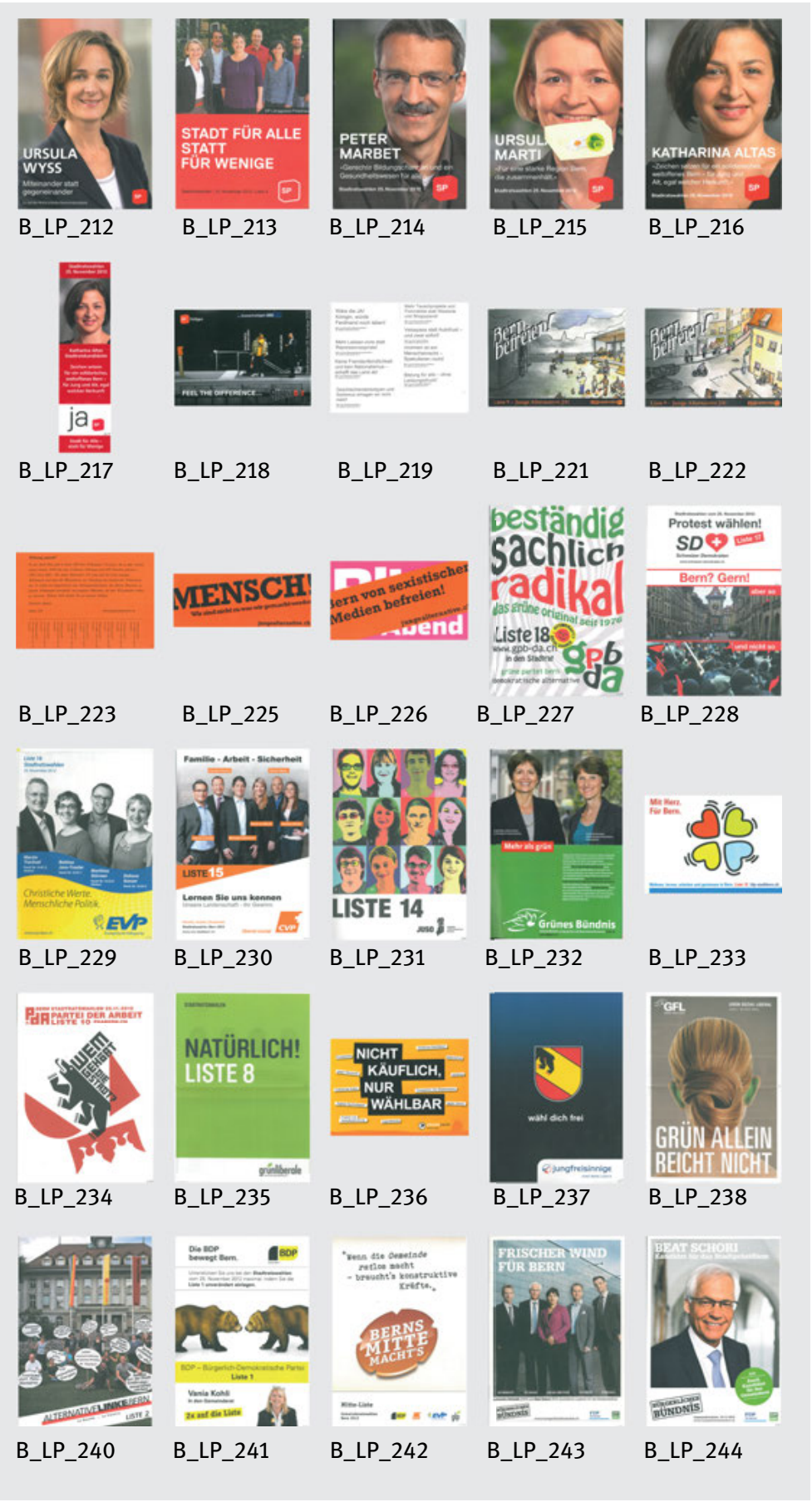




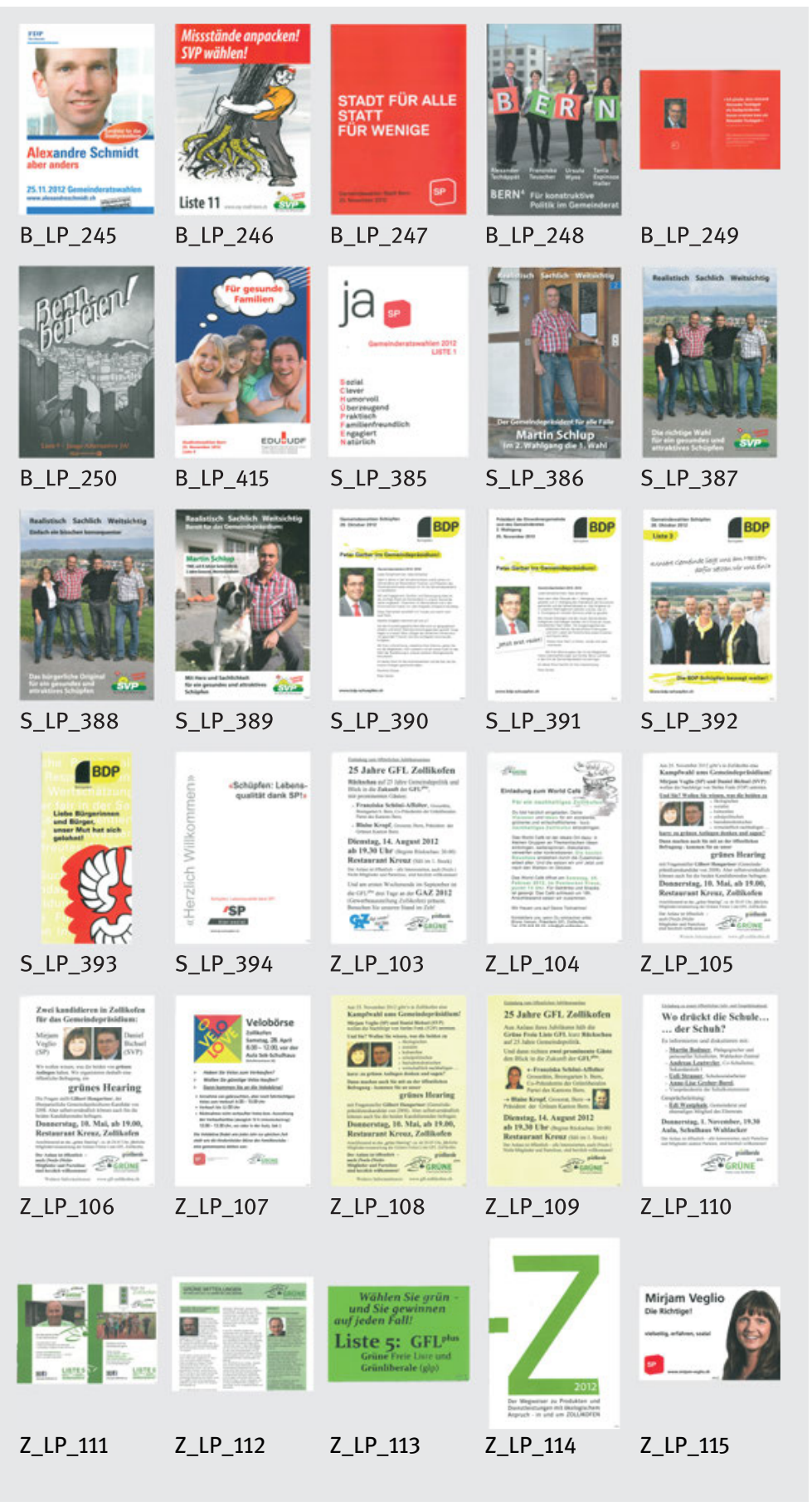




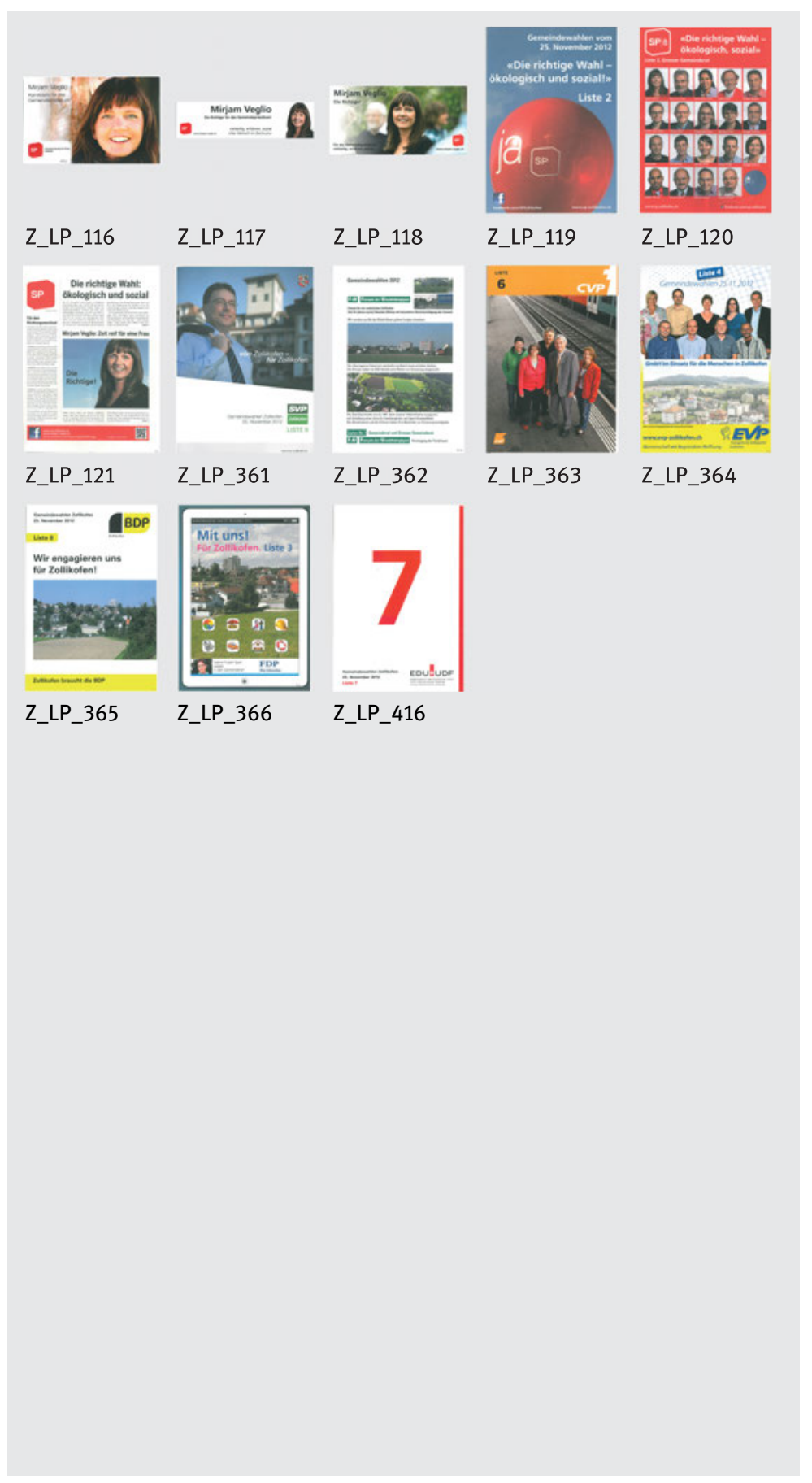




\subsubsection{Gemeinwesenarbeit}

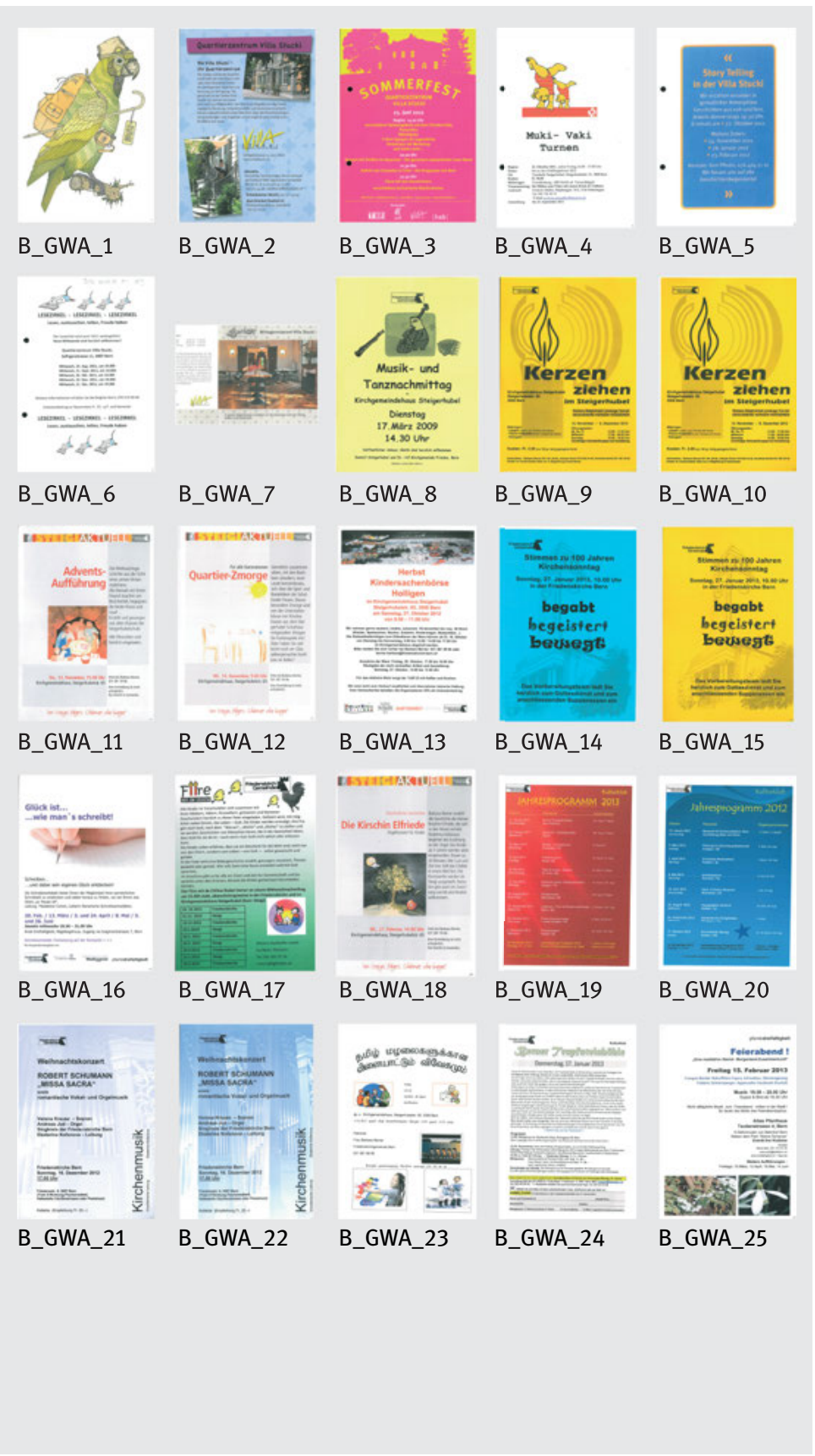




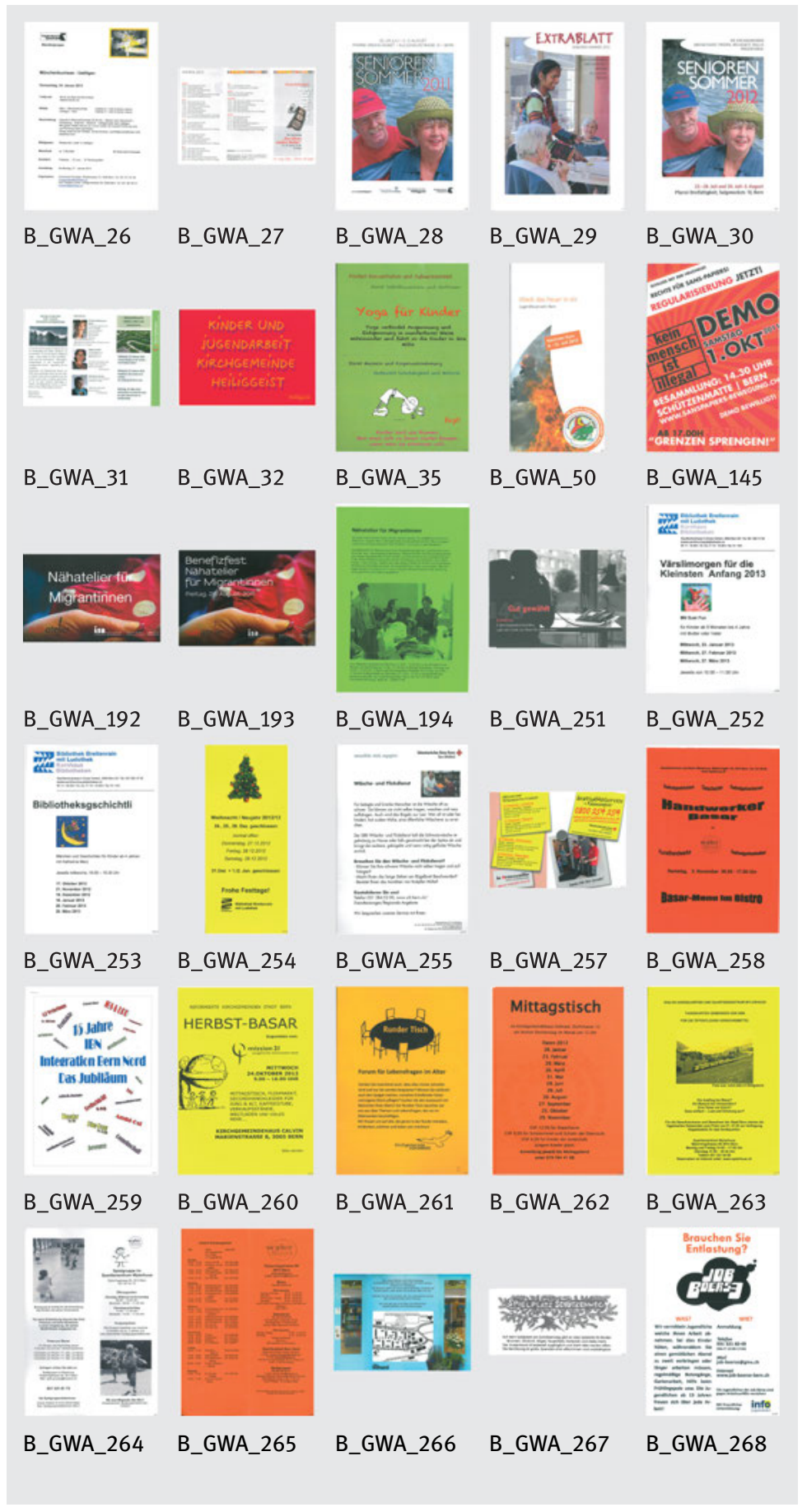




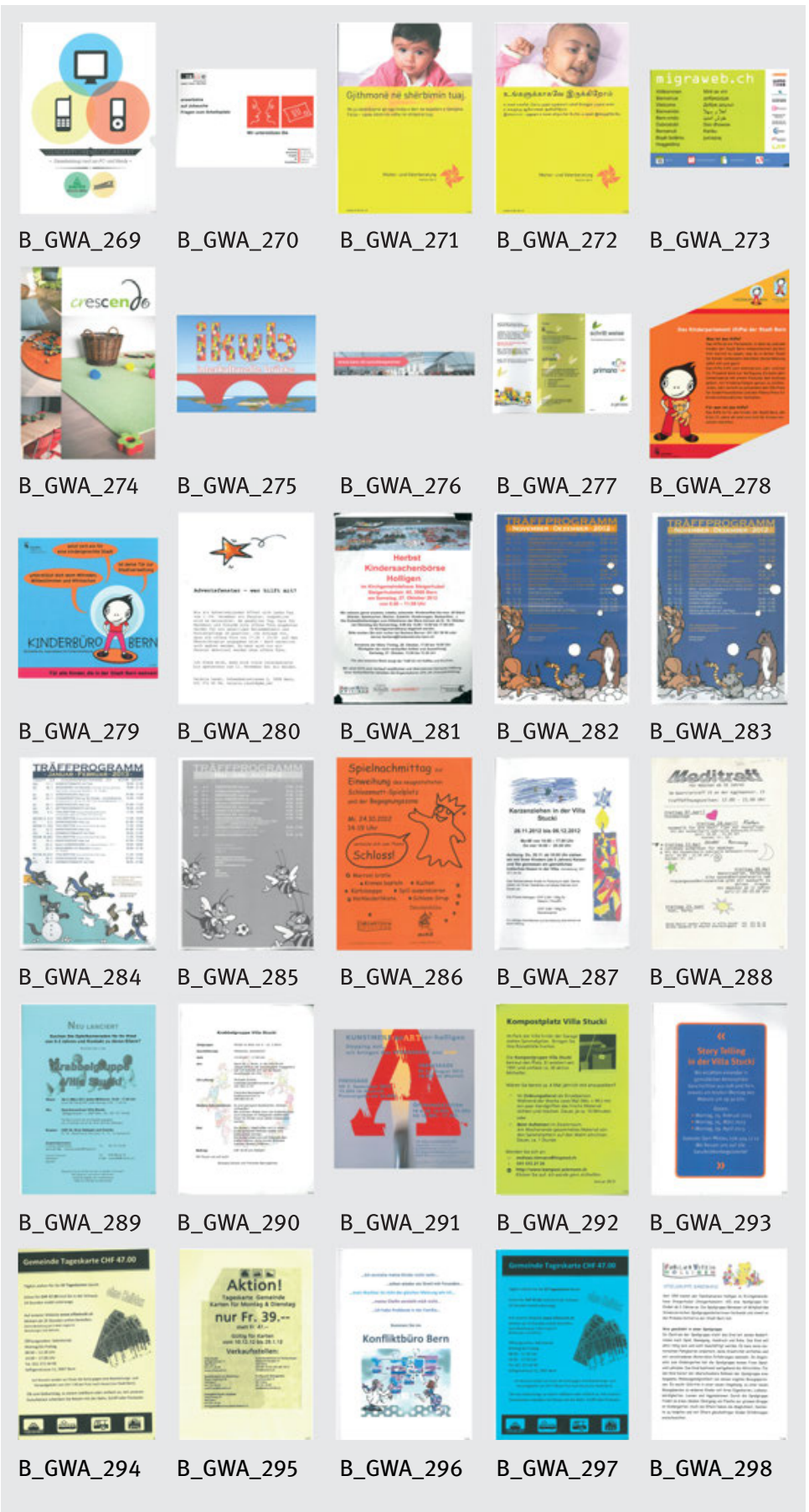




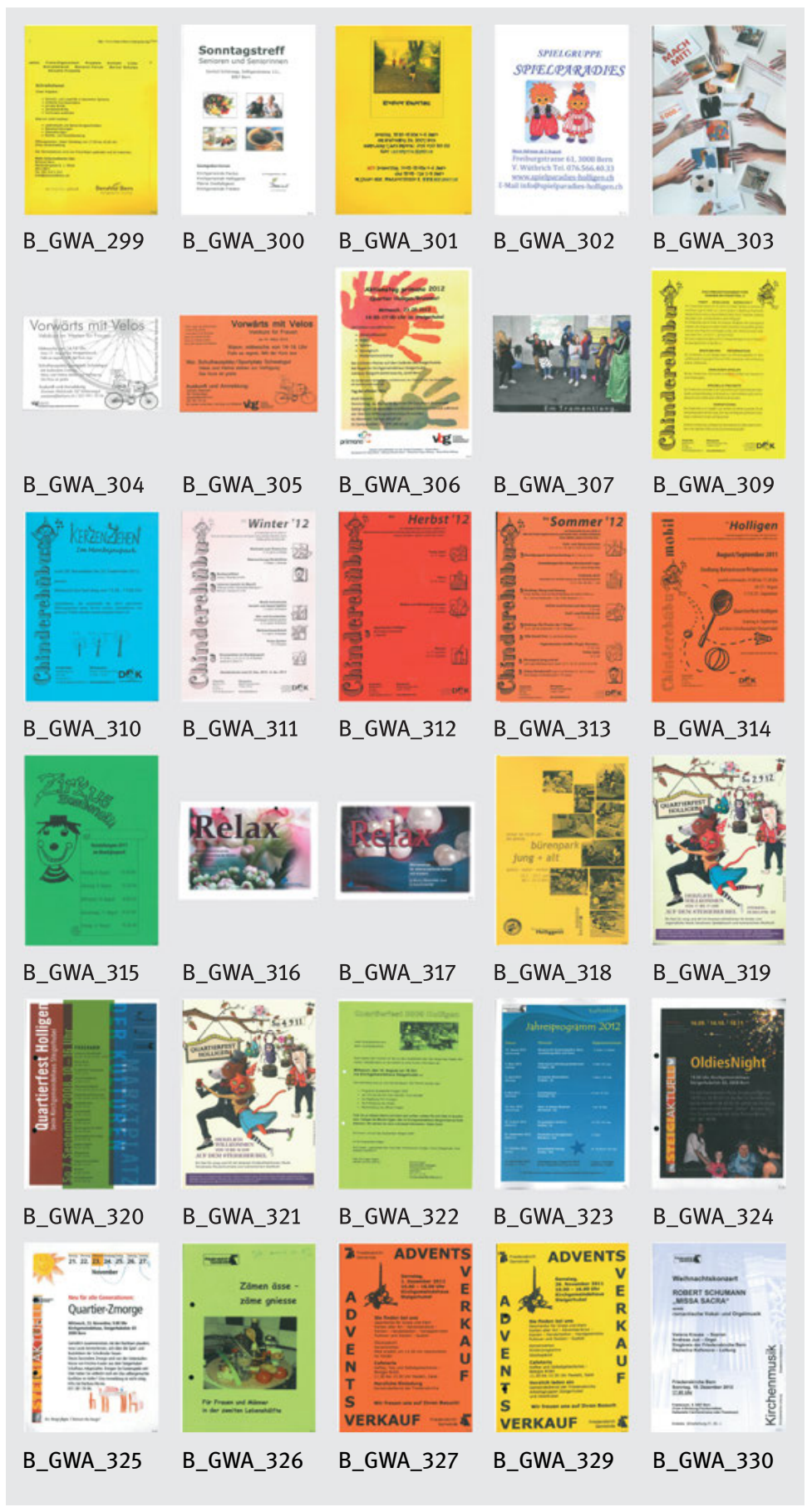




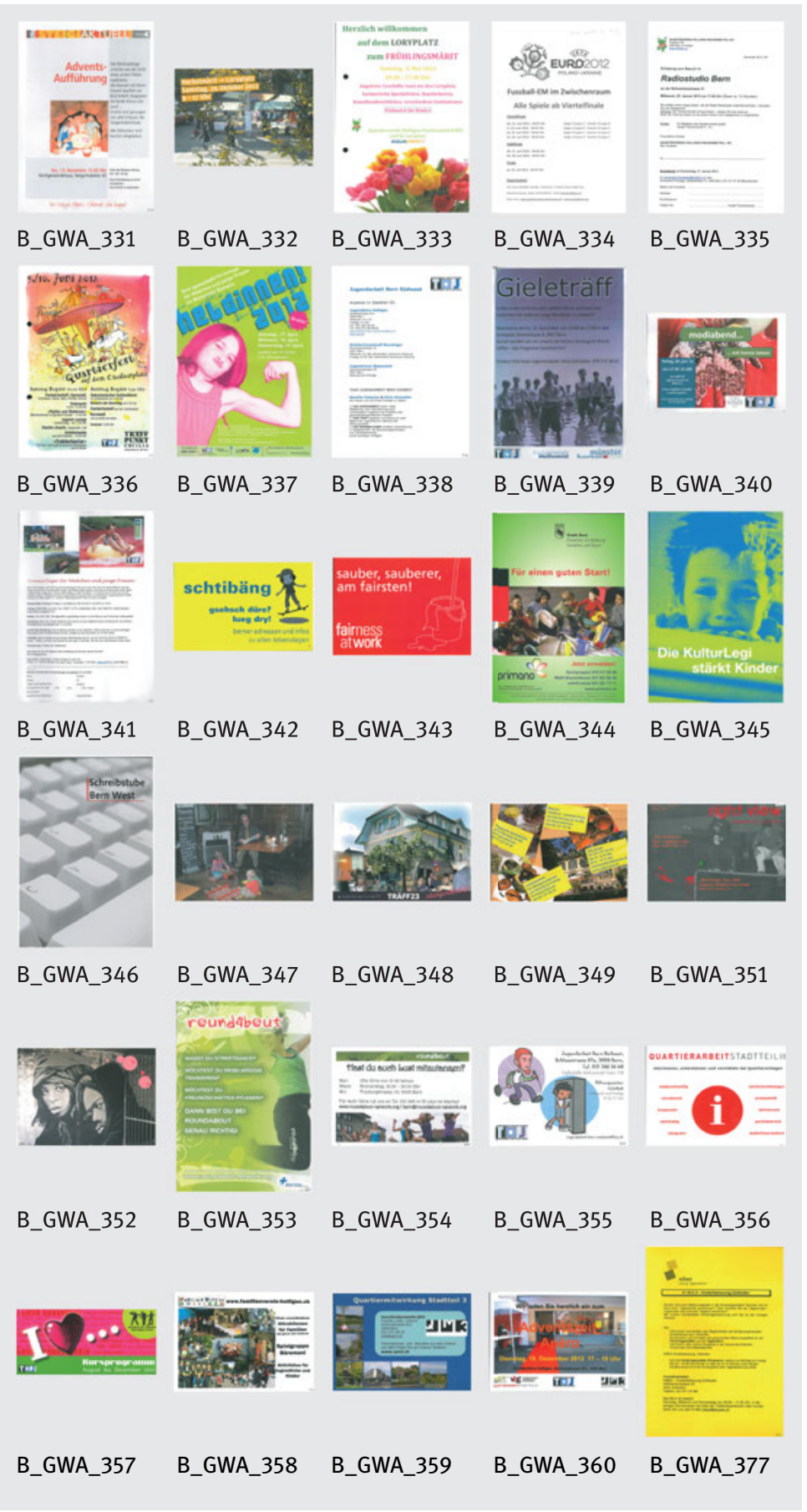




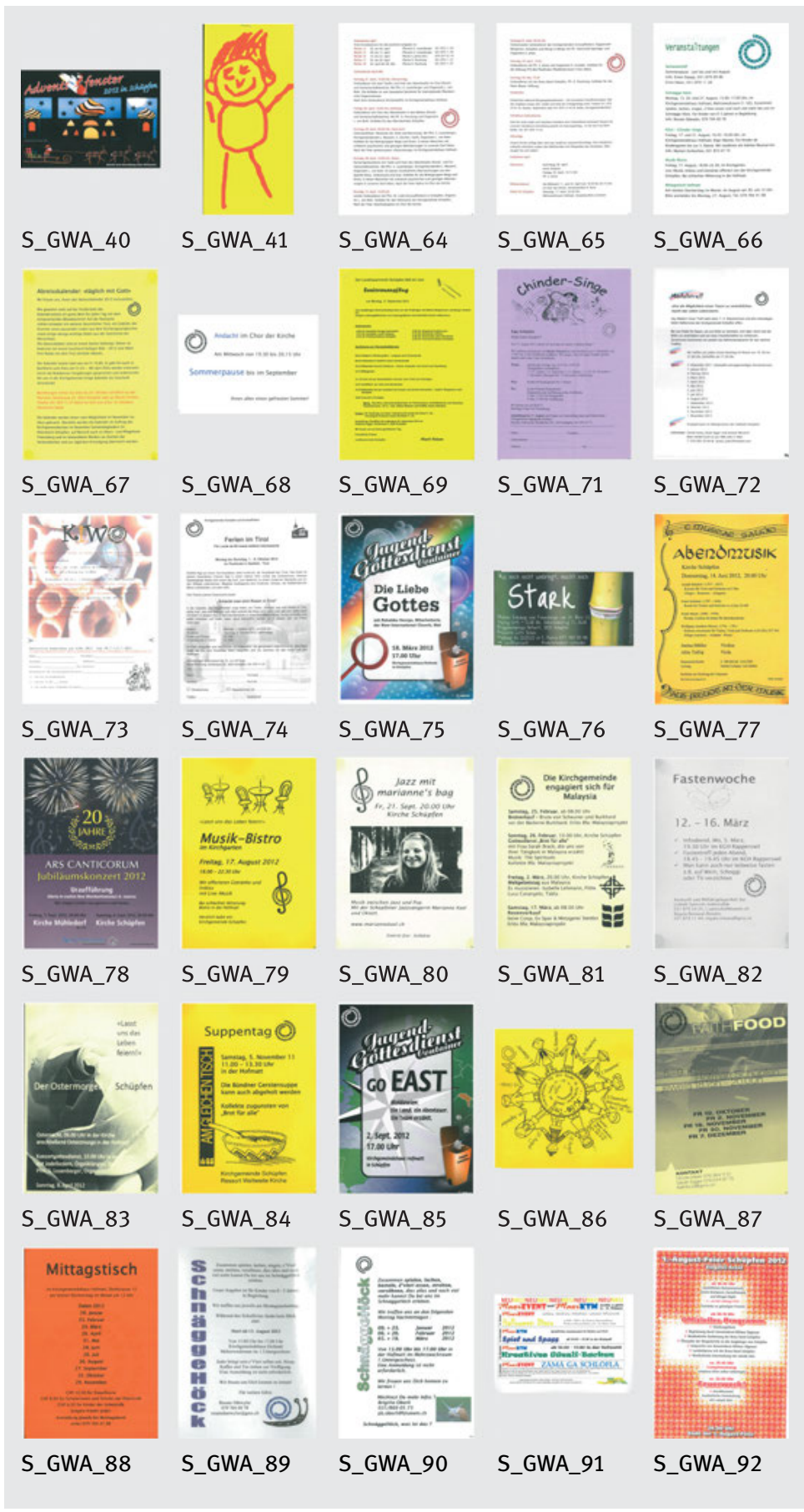




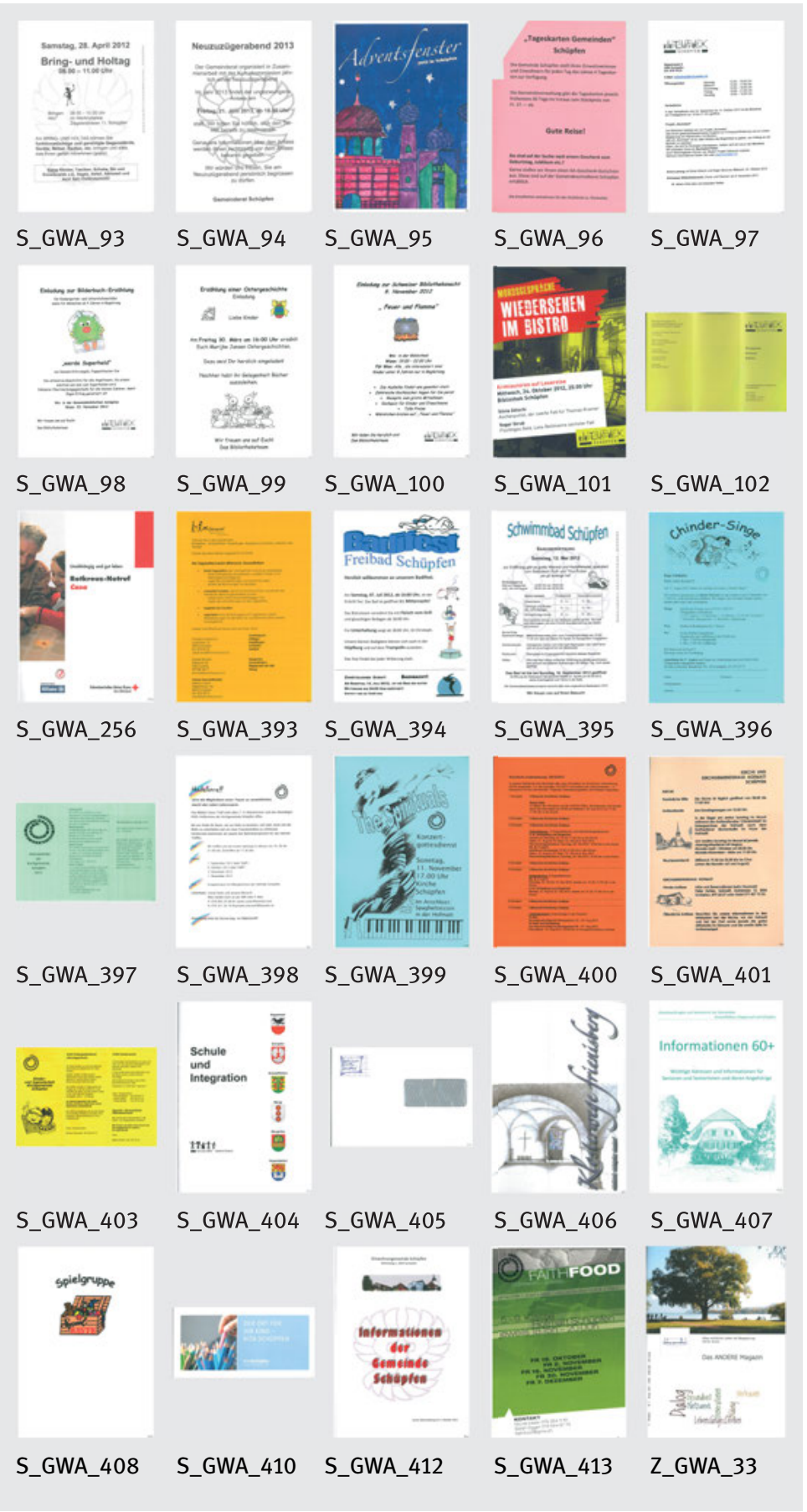




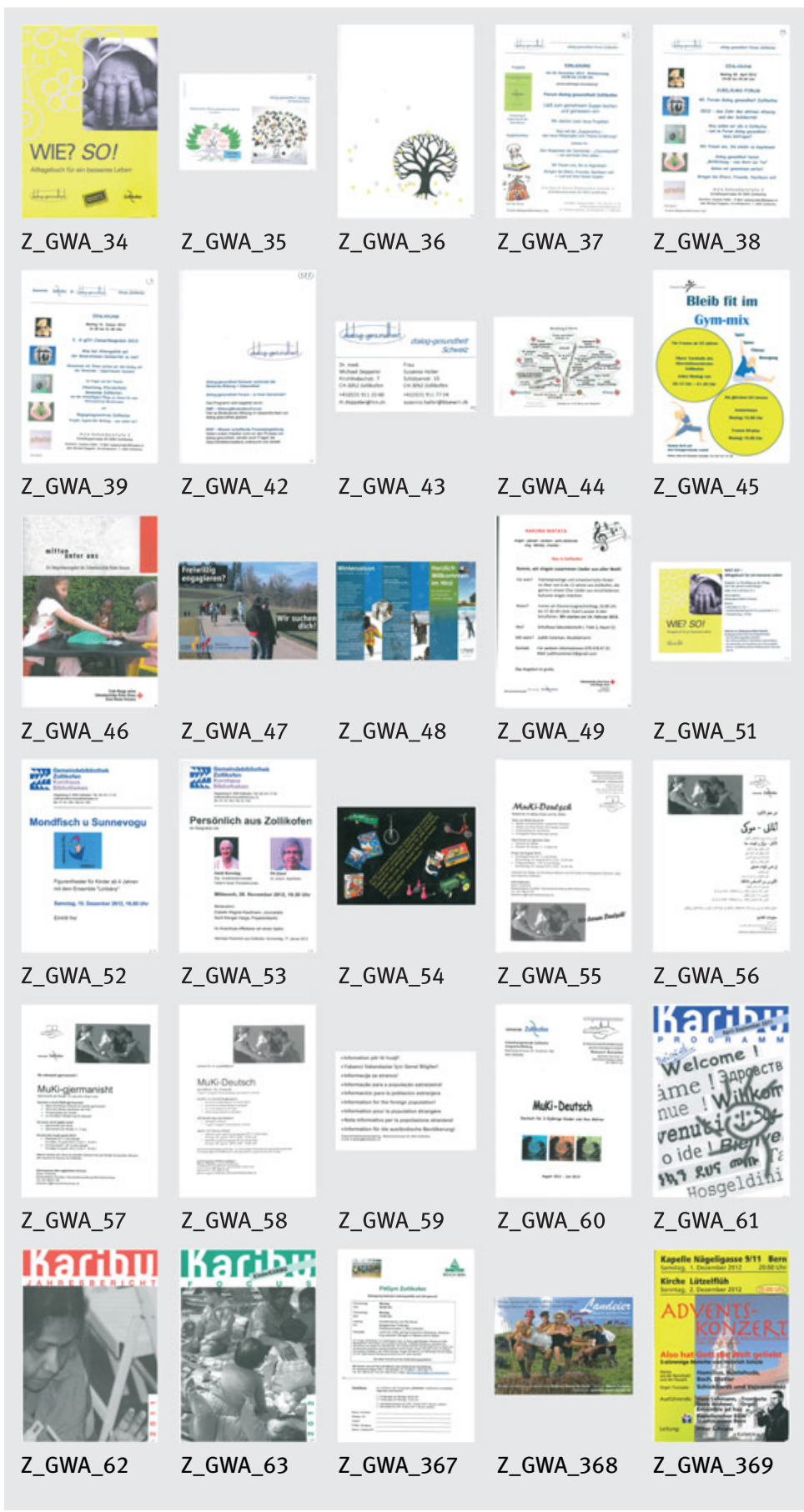




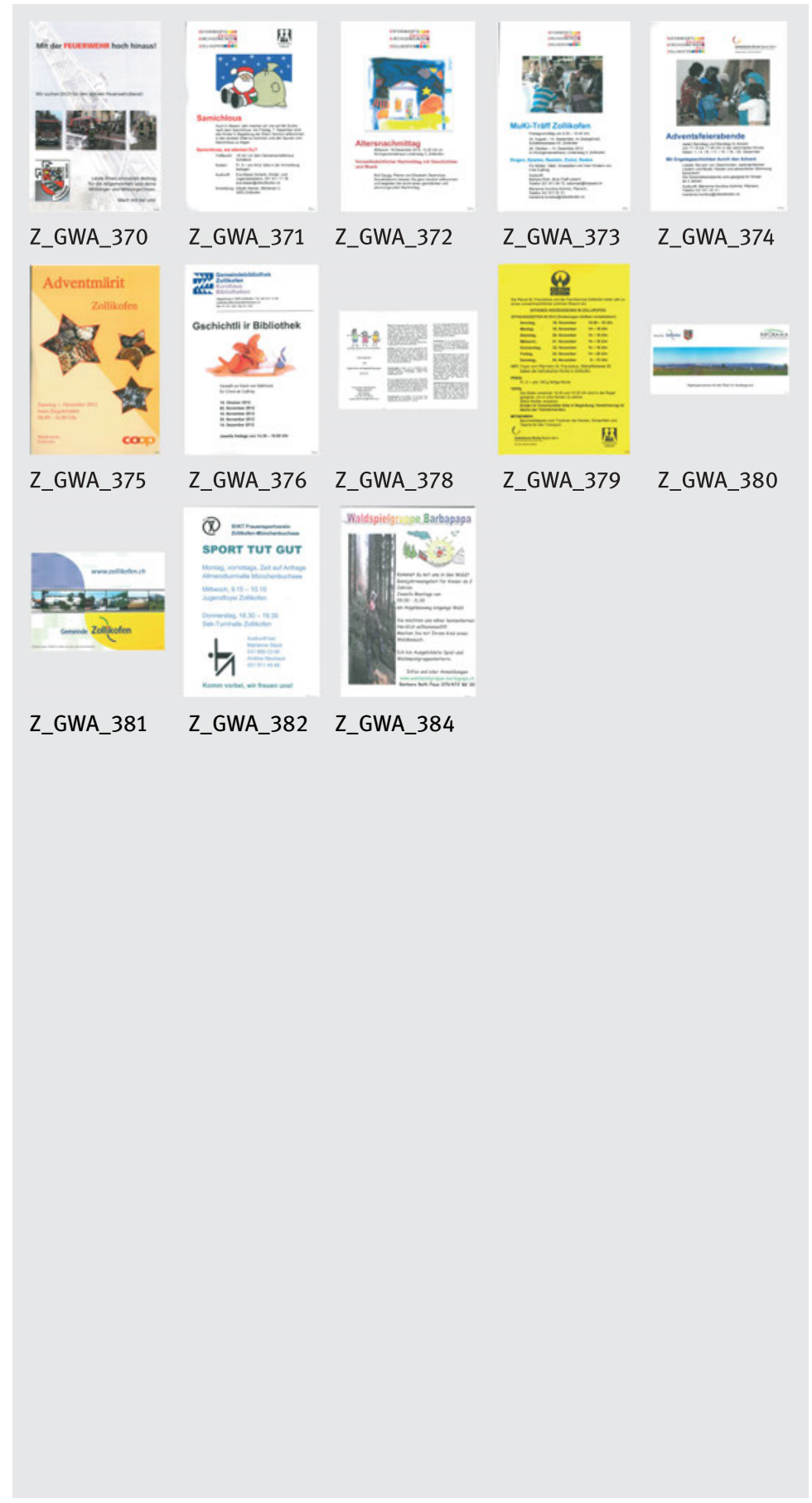




\subsection{Analyseraster Rhetorische Detailanalysen}

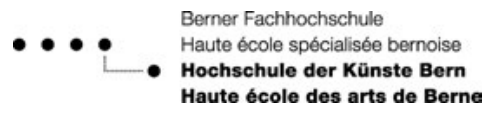

Rhetorische Designanalyse nach dem Berner Modell

Infos und Instruktionen zum Ausfüllen

Beim vorliegenden Material handelt es sich um gesammelte grafische Artefakte aus den Bereichen Gemeinwesenarbeit und Lokalpolitik der Gemeinden Bern, Zollikofen und Schüpfen des Jahres 2012. Sie werden in zwei Schritten von 6 Dozierenden der HKB VisKom und CommDesign individuell analysiert. In einem ersten Schritt haben zwei Dozierende je eine Auslegeordnung des gesamten Materials (rund 400 Flyer, Postkarten, Broschüren, Plakate und Inserate) vorgenommen und eine Auswahl von zwölf repräsentativen Arbeiten für die nun erfolgende detaillierte Rhetorische Designanalyse getroffen.

Für den zweiten Schritt nun bist $D u$ gefragt: Anhand der unten angeführten Leitfragen wirst Du die zwölf Arbeiten analysieren. Für die Detailanalysen wirst du insgesamt vier bis sechs Stunden benötigen (ja nach Detaillierungsgrad deiner Analyse), die vom Projekt vergütet werden. Du kannst frei bestimmen, in welcher Reihenfolge du die Arbeiten analysieren möchtest.

Die Analyse folgt jeweils den immer gleichen fünf Punkten. Es ist dabei hilfreich, wenn Du jeden dieser Punkte so präzise wie möglich beantwortest und dabei jeweils das Auffällige und Typische heraushebst. Du kannst entweder auf dem Computer oder von Hand schreiben - was Dir besser entspricht. Bitte strukturiere Deine Antworten mit Hilfe der im Folgenden verwendeten Nummerierung.

1. Formale Analyse

\begin{tabular}{|c|c|c|}
\hline 1.1 & $\begin{array}{l}\text { Art und Produk- } \\
\text { tion des Artefakts }\end{array}$ & $\begin{array}{l}\text { Broschüre, Faltblatt, Einleger usw. } \\
\text { - Produktionstechnik (z.B. Siebdruck, Prägedruck, Farbkopie, Tin- } \\
\text { tenstrahldrucker, randabfallend) } \\
\text { - Qualität des verwendeten Papiers, Haptik und Veredelung (z.B. } \\
\text { farbiges Kopierpapier, satiniert, niedrige Papiergrammatur, Re- } \\
\text { cyclingpapier) } \\
\text { Textur und ggf. Imitation anderer Materialien durch Papierober- } \\
\text { fläche oder Motive (z.B. metallartig, samtig) }\end{array}$ \\
\hline 1.2 & Format & Postkarte, DIN A4 quer usw. \\
\hline 1.3 & $\begin{array}{l}\text { Eingesetzte } \\
\text { grafische Mittel }\end{array}$ & Foto, Illu, Clipart, Schrift, Logo, Clip-Art, Skizze usw. \\
\hline 1.4 & $\begin{array}{l}\text { Layout, } \\
\text { Gesamtanmutung }\end{array}$ & $\begin{array}{l}\text { Raster, Ausrichtung, formale Anordnung, Proportion und Platzie- } \\
\text { rung (z.B. gleichmässig, chaotisch) } \\
\text { - Form, Verhältnis und Gewichtung der einzelnen Elemente (dia- } \\
\text { gonal, strahlenförmig, symmetrisch, unhierarchisch) } \\
\text { - Gesamtbild, Bild-/Textverhältnis, Vorder-/Hintergrund (z.B. } \\
\text { überfüllt, viel Weissraum, Bild steht im Zentrum, wilder Schrif- } \\
\text { tenmix; Punk, Collage) }\end{array}$ \\
\hline 1.5 & Bildinhalt, Topos & $\begin{array}{l}\text { Verwendete Bildmotive } \\
\text { Vermutete allgemein mit dem Motiv verbundene Symbolik (z.B. } \\
\text { Bildinhalt: Menschen schütteln sich die Hände - Symbolik: Zu- } \\
\text { sammengehörigkeit) }\end{array}$ \\
\hline
\end{tabular}

Gestaltung und Kunst | Arts visuels et design • Konservierung und Restaurierung | Conservation et restauration • Musik | Musique • Oper/Theater | Opéra/ Théâtre • Schweizerisches Literaturinstitut | Institut littéraire suisse $\bullet Y$ Institut | Institut $Y \bullet$ Forschung | Recherche $\bullet$ Weiterbildung | Formation continue 


\begin{tabular}{|c|c|c|}
\hline 1.6 & $\begin{array}{l}\text { Art der Bildgestal- } \\
\text { tung }\end{array}$ & $\begin{array}{l}\text { - Technik, Licht, Perspektive (Portraitfoto, Vogelperspektive, } \\
\text { Weichzeichner, Überbelichtung, Manga, Aquarelltechnik usw.) } \\
\text { - Figur, Muster (Wiederholung, Transformation, Überlagerung, } \\
\text { Kontrast) }\end{array}$ \\
\hline 1.7 & $\begin{array}{l}\text { Typographie } \\
\text { (Mikro/Makro) }\end{array}$ & $\begin{array}{l}\text { - Schriftbild, Typ, Charakter, Grösse, Auszeichnung, Laufweite, } \\
\text { Satz usw. } \\
\text { - bitte sowohl allgemeine Einordnung, z.B. Grotesk, bis hin zu } \\
\text { Schriftart, sofern möglich (z.B. Franklin Gothic Extra Condensed) }\end{array}$ \\
\hline 1.8 & Farbe & $\begin{array}{l}\text { Dominante und sonstig verwendete Farben (auch Papierfarbe } \\
\text { und s/w) } \\
\text { - Farbspektrum, Gesamtbild (z.B. bunte Farbpalette, Regenbogen, } \\
\text { stumpf, gesättigt, dunkle Blautöne, kühle Farben) } \\
\text { - Kontraste (z.B. starker Hell-Dunkel-Kontrast, harmonisch, „beis- } \\
\text { send“) }\end{array}$ \\
\hline 1.9 & $\begin{array}{l}\text { Fehler, } \\
\text { Imperfektion }\end{array}$ & $\begin{array}{l}\text { Welche unbewusst oder mangels Können entstandenen gestalte- } \\
\text { rischen Unschönheiten oder Fehler finden sich? (z.B. verzerrtes } \\
\text { Foto, unregelmässige Spationierung, mangelnde Hierarchie) } \\
\text { - Gibt es Imperfektionen, die ev. bewusst eingesetzt wurden? }\end{array}$ \\
\hline
\end{tabular}

2. Wirkungsanalyse

\begin{tabular}{|c|c|c|}
\hline 2.1 & Gesamteindruck & $\begin{array}{l}\text { Bitte schildere spontan deinen ersten Eindruck, deine allgemeine } \\
\text { Einschätzung, persönliche Bewertung. }\end{array}$ \\
\hline 2.2 & Einzelwirkungen & $\begin{array}{l}\text { Kannst du Wirkungen benennen, die das Artefakt auf dich hat? } \\
\text { - Hier kannst du sowohl persönliche, subjektive oder wertende } \\
\text { Wirkungen nennen (z.B. witzig, langweilig, sympathisch, nichts- } \\
\text { sagend, auffällig, seriös), die fachterminologisch nicht präzise } \\
\text { sein müssen (schön, grässlich, billig, rührend etc.) wie auch Wir- } \\
\text { kungen, die du der Arbeit aufgrund deines professionellen Hin- } \\
\text { tergrunds zuschreibst (z.B. harmonisch, dilettantisch, inszeniert, } \\
\text { inspiriert, lesbar, unübersichtlich). }\end{array}$ \\
\hline 2.3 & $\begin{array}{l}\text { Wirkungs- } \\
\text { dimensionen } \\
\text { logos } \\
\text { ethos } \\
\text { pathos }\end{array}$ & $\begin{array}{l}\text { Bitte beschreibe, auf welchen Wirkungsebenen sich die genann- } \\
\text { ten Wirkungen entfalten: Sind sie auf der Verstandesebene anzu- } \\
\text { siedeln (logos), vermitteln oder verändern sie Werte oder sind } \\
\text { Ausdruck für den Charakter des Absenders (ethos) oder spielen } \\
\text { sie sich auf der Gefühls-, Affekt- oder Emotionsebene ab (pa- } \\
\text { thos)? } \\
\text { - Mehrere Wirkungs dimensionen pro Artefakt sind möglich. }\end{array}$ \\
\hline 2.4 & $\begin{array}{l}\text { Stilhöhe } \\
\text { niedrig } \\
\text { mittel } \\
\text { hoch }\end{array}$ & $\begin{array}{l}\text { Auf welcher Stilhöhe würdest du die Arbeit einordnen? Ist der } \\
\text { Stil eher niedrig, d.h. schlicht, sachlich und nüchtern, oder ist er } \\
\text { hoch, d.h. affektstark, wurde mit intensiven visuellen Mitteln ge- } \\
\text { arbeitet? Oder ist die Stilhöhe irgendwo dazwischen anzusiedeln } \\
\text { (mittlerer Stil)? } \\
\text { - Mit welchen Stilmitteln verbindest du die von dir zugeschriebe- } \\
\text { ne Stilhöhe konkret? }\end{array}$ \\
\hline 2.5 & Wirkungsskalen & - Wo auf den vier Wirkungsskalen (s. separates Blatt) würdest du \\
\hline
\end{tabular}




\begin{tabular}{|l|l|l|}
\hline & $\begin{array}{l}\text { die Arbeit einstufen? Welcher Begriff trifft zu? } \\
\text { Grün angemessen }\end{array}$ & $\begin{array}{l}\text { Klebe einen Klebepunkt an diese Stelle oder zeichne den Begriff } \\
\text { farbig an. }\end{array}$ \\
$\begin{array}{l}\text { Gelb in Frage } \\
\text { kommend }\end{array}$ & $\begin{array}{l}\text { Die gewählte Farbe sagt aus, ob du diese Wirkung in Bezug auf } \\
\text { den Kontext (Inhalt, Anlass, Zielpublikum, Absender etc.) des Ar- } \\
\text { Rot nicht ange- } \\
\text { messen }\end{array}$ & \begin{tabular}{l} 
tefakts für angemessen hältst. \\
\hline
\end{tabular} \\
\hline
\end{tabular}

\section{Wirkregeln}

Hier interessiert uns das Zusammenspiel von Stilmittel und Wirkung:

- Siehst du Zusammenhänge zwischen formalen Merkmalen des Artefakts und seinen Wirkungen (z.B. „Die unruhige Wirkung kommt durch die Mischung verschiedener Schriften und die diagonale Anordnung zustande")?

- Kannst du sie auf Wirkregeln zurückführen, die dir bekannt sind? (z.B. „Leichtigkeit durch viel Weissraum“, „Schwarz = elegant" etc.)

- Gibt es Wirkungen, die du nicht erklären kannst?

4. Angemessenheit

- Welche Absichten vermutest du hinter dieser Arbeit? Wer soll angesprochen werden? Welche Wirkungen sollten angesichts des Kontexts vermutlich erzielt werden?

- Was an dieser Arbeit funktioniert deiner Meinung nach „im Sinne des Erfinders“, was nicht? Oder anders gefragt: Was wurde wohl so nicht beabsichtigt?

- Inwiefern hältst du diese Arbeit für angemessen, zweckmässig oder gar gelungen? Was ist unpassend und warum? Was wäre verbesserungswürdig (und wie)?

5. Besonderes

- Gibt es Elemente, die dir typisch oder untypisch für diese Art von Gestaltung erscheinen?

- Was fällt dir Besonderes auf, das im Vorherigen noch nicht erwähnt wurde oder im Vergleich mit den anderen analysierten Arbeiten? 


\subsection{Leitfaden und Fragebogen Wirkungsstudie}

\section{Befragung Designvarianten RTD}

Projekt Amateurgestaltung

1. Leitfaden Befragung zu Gestaltung und Wirkung der Varianten

1. Was ist Ihr erster Eindruck, wenn Sie diesen Flyer/Prospekt sehen?

2. Wie wirkt er konkret auf Sie?

3. Auf welche Gestaltungsmerkmale führen Sie die Wirkung zurück?

4. Wird für Sie rasch klar, wofür diese Flyer/Prospekt wirbt oder um welches Angebot es geht?

5. Ist dieser Flyer/Prospekt Ihrer Meinung nach professionell gestaltet?

6. Halten Sie diesen Flyer/Prospekt für angemessen gestaltet?

Diese 6 Fragen werden zu allen 9 Designvarianten (inkl. Originale) gestellt, wobei die Reihenfolge variiert wird. Durch Nachfrage können die Antworten spezifiziert werden (z.B. Warum war für Sie nicht auf Anhieb erkenntlich, um welches Angebot es geht? Warum halten Sie den Flyer für unprofessionell gestaltet?).

Sobald die drei Varianten eines Bereichs beurteilt wurden, werden diese miteinander verglichen (auch hier allenfalls Begründung erfragen):

7. Wenn Sie die drei Varianten miteinander vergleichen, welche ist für Sie die passendste, welche passt am wenigsten?

8. Welche ist für Sie am professionellsten gestaltet, welche am wenigsten professionell?

Zum Schluss werden die soziodemografischen Daten erhoben sowie die Nähe der Befragten zu den beworbenen Absendern oder Angeboten.

2. Soziodemographische Daten

Geschlecht: w $\square \quad \mathrm{m} \square$

Alter:

Ausbildung:

Beruf:

Wohnort:

Nationalität: 


\section{Erhebung Politische Haltung und Nähe zum Absender oder Angebot}

\section{Wahlflyer BDP}

1. Wie nahe stehen Sie politisch der BDP?
Sehr nahe
eher nahe
indifferent
eher entfernt
ganz entfernt
$\square$
$\square$
$\square$

2. Könnten Sie sich vorstellen, Kandidierende der BDP zu wählen?
Ja
nein
vielleicht
$\square$
$\square$

\section{Moditreff}

1. Für wie sinnvoll halten Sie grundsätzlich das Angebot eines Mädchentreffs?

Sehr sinnvoll eher sinnvoll indifferent eher sinnlos sinnlos

$\square$

$\square$

2. Könnten Sie sich vorstellen, Ihre Tochter an einem Mädchentreff teilnehmen zu lassen?
Ja nein vielleicht
$\square \quad \square$

\section{Freiwillige Feuerwehr}

1. Für wie sinnvoll halten Sie das Angebot der freiwilligen Feuerwehr? Sehr sinnvoll eher sinnvoll indifferent eher sinnlos sinnlos $\square$

$\square$ $\square$

2. Könnten Sie sich vorstellen, selbst bei der freiwilligen Feuerwehr mitzumachen?
Ja nein vielleicht
$\square \quad \square \quad \square$ 
